\title{
Flexural Strength of an Indirect Composite Modified with Single-Wall Carbon Nanotubes
}

\author{
Pedro Rogério Camargos Pennisi ${ }^{1}$ Pedro Urquiza Jayme Silva ${ }^{1}$ Fábio Scorsolini Valverde² \\ Ticiane Campos Clemente ${ }^{2}$ Vitória Cerri ${ }^{2}$ Maria Eduarda Biaco ${ }^{2}$ Rebeca Garcia Rosa Ferreira ${ }^{3}$ \\ Luiz Renato Paranhos ${ }^{1}$ Eduardo Buozi Moffa ${ }^{4}$
}

1 School of Dentistry, Federal University of Uberlândia, Uberlândia/ MG, Brazil

2 School of Dentistry, University Center - UNIFAE, São João da Boa

Address for correspondence Eduardo Buozi Moffa, DDS, MSc, PhD, UNIFAE School of Dentistry, Largo Engenheiro Paulo de Almeida Sandeville $\mathrm{N}^{\circ} 15$, São João da Boa Vista-Sao Paolo, Zip code:

Vista/Sao Paolo, Brazil

35chool of Physiotherapy, University Center - UNIFAE, São João da 13.870-377, Brazil (e-mail: eduardo.moffa@prof.fae.br).

\begin{abstract}
Objectives The low resistance to fracture has limited the use of indirect composite resins for dental restorations, particularly in regions that are exposed to strong occlusal forces. To overcome this issue, different types of reinforcement for composites have been proposed, one of which is carbon nanotubes (CNTs). The aim of this study was to evaluate the flexural resistance of one commercial indirect composite resin (Sinfony, 3M/ESPE) after incorporation of single-wall carbon nanotubes (SWCNTs; Sigma-Aldrich, Inc., St. Louis, Missouri, United States) with or without the silanization form.

Materials and Methods Specimens of composite resin were fabricated in a Teflon mold. The composite resin was prepared according to the manufacturer's instructions ( $n=10$ for each group), with SWCNTs in three concentrations.

Statistical Analysis The SWCNTs and $\mathrm{SWCNT} / \mathrm{SiO}_{2}$-ATES specimens were evaluated by transmission electron microscopy, and a flexural test was conducted according to the ISO 4049/2009. Flexural strength data in MPa were submitted to one-way ANOVA following Tukey $(p<0.05)$.

Results The SWCNTs did not improve the flexural strength of indirect composite resin when compared with the control, independent of the concentration added $(p>0.05)$. However, when SWCNTs and SWCNTs/SiO2-ATES were compared, the SWCNTs/SiO2-

Keywords

- carbon nanotubes

- composite resins

- flexural strength

ATES showed higher values than the three concentrations of SWCNTs $(p<0.05)$.

Conclusion The silanization process improves the SWCNTs strength proprieties, but the modification of chemical bonding between SWCNT and SWCNT/SiO ${ }_{2}$-ATES modified resins, in different concentrations, did not improve the composite resin flexural strength.
\end{abstract}

\section{Introduction}

Improvements in the properties of indirect composite resins (ICR) have been used in applications for inlays, onlays, and crowns. ${ }^{1}$ Despite the significant development of these composites, ICR have shown unsatisfactory clinical performance, particularly in regions exposed to strong mastication
DOI https://doi.org/ $10.1055 / \mathrm{s}-0040-1721315$ ISSN 1305-7456.

\section{(C) 2020. European Journal of Dentistry.}

This is an open access article published by Thieme under the terms of the Creative Commons Attribution-NonDerivative-NonCommercial-License, permitting copying and reproduction so long as the original work is given appropriate credit. Contents may not be used for commercial purposes, or adapted, remixed, transformed or built upon. (https://creativecommons.org/licenses/by-nc-nd/4.0/) Thieme Medical and Scientific Publishers Pvt. Ltd., A-12, 2nd Floor, Sector 2, Noida-201301 UP, Indiay 
forces, ${ }^{2-4}$ and their relatively low resistance to fracture has limited the clinical usage. ${ }^{4,5}$

A common technique to improve composite mechanical properties is the incorporation of fibers. ${ }^{6}$ Different types of reinforcement for composites proposed have included carbon, graphite, glass, and Kevlar. ${ }^{78}$ The effectiveness of fiber reinforcement is dependent on many variables: materials used, the quantity of fibers in the matrix, diameter, length, orientation of the fibers, and adhesion of fibers to the matrix polymer. ${ }^{9-1011}$ Some laboratory studies demonstrated that these reinforced resins had improved resistance to fracture, ${ }^{12,13}$ while other studies have shown that there was no significant increased resistance to fracture. ${ }^{14,15}$

The carbon nanotubes (CNTs) have excellent mechanical properties, electrical conductivity, rheological properties, thermal conductivity, stability, and flammability. ${ }^{16}$ Therefore, the incorporation of CNTs can significantly enhance of such properties of polymer composite. ${ }^{17}$

Various applications of CNTs fabrication of reinforced fibers and nanocomposites have been demonstrated in literature. ${ }^{18}$ There are two types of CNT: single-wall carbon nanotubes (SWCNTs) and multi-wall carbon nanotubes (MWCNTs), ${ }^{16,19}$ and various properties of these CNTs have been published. Single and multi-wall CNT vary significantly depending on the production, purification, suspension, filling, and functionalization. ${ }^{19}$ In addition, good interfacial bonding between polymer and CNTs and the uniform dispersion of CNTs throughout the polymer matrix are critical in determining the mechanical properties of polymer CNTs composites. ${ }^{20}$ According to the above considerations, the CNTs can be an excellent candidate to be incorporated in the laboratory-processed resin composite ${ }^{16}$ to improve their flexural resistance, although there are no studies on this issue. Therefore, the purpose of this study was evaluated the flexural resistance of one commercial composite resin after incorporation of CNTs. Also, the effect of the silanization of CNTs on flexural resistance was studied. It is expected that a good dispersion and bonding of SWCNTs to dental resin can be achieved improving the mechanical properties.

\section{Materials and Methods}

\section{Ethical Criteria}

The present study followed the Krithikadatta et $\mathrm{al}^{21}$ guidelines that suggests the elaboration of Checklist for Reporting In vitro Study Guidelines standardizing in vitro studies. This study did not require approval of ethical criteria, as it did not involve humans and animals directly and indirectly.

\section{Sample Qualification and Sample Calculation}

In this study, the sample size was calculated based on previous studies ${ }^{22}$ with a statistical power of 0.80 , requiring 10 specimens per group.

The CNTs used were SWCNTs (Sigma-Aldrich, Inc.; St. Louis, Missouri, United States). The specimens were allocated in five groups to be analyzed ( - Fig. 1): control group

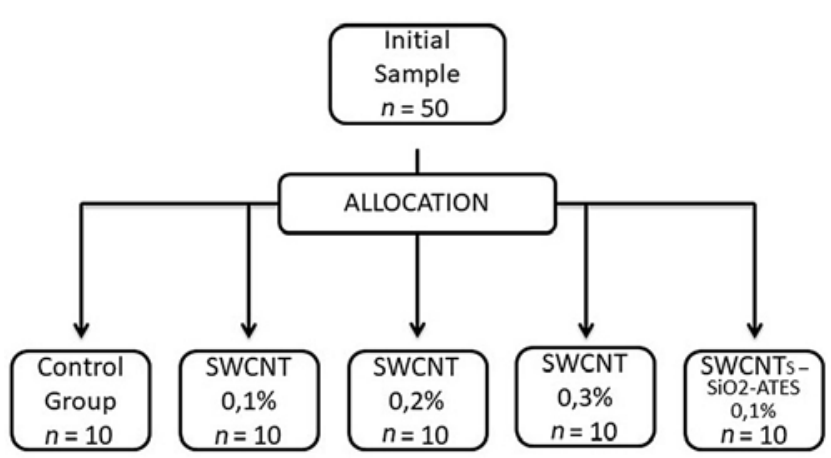

Fig. 1 Allocation process flow chart.

(without SWCNTs), SWCNTs at $0.1 \%$, SWCNTs at $0.2 \%$, SWCNTs at $0.3 \%$, and $\mathrm{SWCNTs}-\mathrm{SiO}_{2}$-ATES at $0.1 \%$.

\section{Silanization of Carbon Nanotubes}

The silanization methodology followed Zhang et al. ${ }^{22}$ First, the coating of Nano-SiO ${ }_{2}$ on CNT was prepared. This process involved sonication of SWNTs in an ethanol solution for 30 minutes, then incubation with $5 \mathrm{mmol} / \mathrm{L}$ APTES (3-aminopropyltriethoxysilane) for 30 minutes. The APTEStreated SWNTs were washed thoroughly with ethanol and were ultrasonically scattered in $20 \mathrm{~mL}$ ethanol for 30 minutes. Subsequently, $200 \mu \mathrm{L}$ of concentrated $\mathrm{NH}_{3} \cdot \mathrm{H}_{2} \mathrm{O}$ and $100 \mu \mathrm{L}$ of TEOS (tetraethylorthosilicate) were added. After 30 minutes another $100 \mu \mathrm{L}$ of TEOS was added. For the desired $\mathrm{SiO}_{2}$ nanocoated SWNTs product the hydrolyzation process was conducted in ultrasonic conditions for $90 \mathrm{~min}$. The samples were separated and washed with ethanol. The SWNTs-SiO ${ }_{2}$ were surface modificated with $5 \mathrm{mmol} / \mathrm{L}$ of organosilane ATES (allyltriethoxysilane) in ethanol, to produce the SWCNT-SiO 2 -ATES. After washing with ethanol, the sample was dried at $120^{\circ} \mathrm{C}$ and cooled in a desiccator.

\section{Incorporation of Carbon Nanotubes in the Composite Resin}

The composite resin was prepared according to fabricant instructions, without modifications for control group (without SWCNTs), and SWCNTs were added at $0.1,0.2$, and $0.3 \%$ $(\mathrm{w} / \mathrm{w})$ and $\mathrm{SWCNTs}-\mathrm{SiO}_{2}$-ATES at $0.1 \%(\mathrm{w} / \mathrm{w})$. The resins were mechanically blended by spatulation to mimic clinical preparation.

After incorporations of SWCNTs and SWCNTs-SiO ${ }_{2}^{-}$ ATES in different concentrations, specimens were fabricated in a Teflon mold $(25 \times 2 \times 2 \mathrm{~mm})$, prepolymerized using a visible-light-curing for 5 seconds with the Visio Alfa, and then polymerized in the Visio Beta device for 16 minutes under vacuum. All polymerization devices were used according to the manufacturer's instructions (3M/ ESPE). Wet silicon carbide papers (600-, 800-, 1,000-, and 1,500-grit) were used to polish and achieve a thickness of $2 \mathrm{~mm}$; that was confirmed with a digital micrometer (Mitutoyo Corp.; Tokyo, Japan). The specimens were bathed in distilled water at $37^{\circ} \mathrm{C}$ for 24 hours. 


\section{Data Collection and Analysis}

The SWCNTs and SWCNT/SiO 2 -ATES specimens were evaluated by transmission electron microscopy (TEM), and the flexural test was conducted according to the ISO 4049/2009 (ISO-Standards, 2009) performed in an EMIC DL 2000 assay machine connected to a 1,000 kgf loading cell with a speed of $0.5 \mathrm{~mm} /$ minute. The flexural strength was calculated using the formula:

$$
F=3 P f L / 2 W H^{2}
$$

where $F$ is flexural strength, $P f$ is the maximum load $(\mathrm{N})$, $L$ is the distance between supports ( $\mathrm{mm}), W$ is the sample width ( $\mathrm{mm}$ ), and $H$ is the height of the sample ( $\mathrm{mm}$ ). Flexural strength data in MPa were submitted to analysis of variance (one-way ANOVA), following Tukey $(p<0.05)$.

\section{Results}

As refering to the flexural strength data in - Table 1, no significant difference was found among SWCNTs/0.1, SWCNTs/0.2, and SWCNTs/0.3 groups, comparing to the control group. The results also revealed that the SWCNTs/SiO2-ATES samples showed higher flexural strength than the others experimental groups, with the exception of the unmodified control resin, which exhibited the highest values. The TEM image of

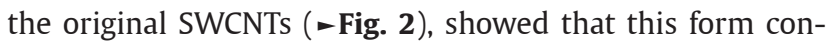
tained many impurities, mainly amorphous carbon and metal nanoparticles. The TEM image of SWCNTs after the silanization treatment ( $\boldsymbol{- F i g .} \mathbf{3}$ ) showed that the impurities were almost completely removed, and the dispersion was also improved. The TEM image of SWCNTs/SiO2-ATES also reveals the presence of many spherical nanoparticles that form a thin shell on the outer surface of the SWCNTs.

\section{Discussion}

In this study, the flexural strength was evaluated to determine the efficacy of indirect composite reinforcement of Sinfony by SWCNTs with two chemical surface modifications. Two main problems have to be solved to effectively improve the material properties of composite by adding CNT as reinforcement. These problems are the interfacial bonding and the dispersion of CNTs in the polymeric matrix. CNTs are an inherently inert material and easy to agglomerate. In addition, slipping in the bundle and weak interface bonding result in inefficient load transfer, decreasing the mechanical properties of CNT reinforced composite. Thus, the particles need some surface modification to reduce clustering and improve their dispersion throughout the composite matrix. ${ }^{23}$ This process involves attachment of functional groups to nanotubes walls or covalent attachment. ${ }^{22}$

- Table 1 shows that the composite containing SWCNTs/ SiO2-ATES exhibited higher flexural strength than that of original SWCNTs content $0.1,0.2$, and $0.3 \mathrm{wt} \%$. This lower

Table 1 Means and standard deviations of the flexural strength(MPa)

\begin{tabular}{|l|l|l|l|l|l|}
\hline Group & CG & $\begin{array}{l}\text { SWCNTs/ } \\
\mathrm{SiO}_{2} \text {-ATES }\end{array}$ & SWCNTs/0.1 & SWCNTs/0.2 & SWCNTs/0.3 \\
\hline Mean & 100.35 & 91.58 & 85.24 & 84.08 & 83.36 \\
\hline SD & 1.25 & 1.35 & 1.05 & 2.04 & 2.59 \\
\hline & $\mathrm{A}$ & $\mathrm{B}$ & $\mathrm{C}$ & $\mathrm{C}$ & $\mathrm{C}$ \\
\hline
\end{tabular}

Abbreviations: SWCNT, single-wall carbon nanotube; CG, Corega group; SD, standard deviation.

Note: Horizontally, mean values designated with identical capital letters were not statistically different $(p>0.05)$.

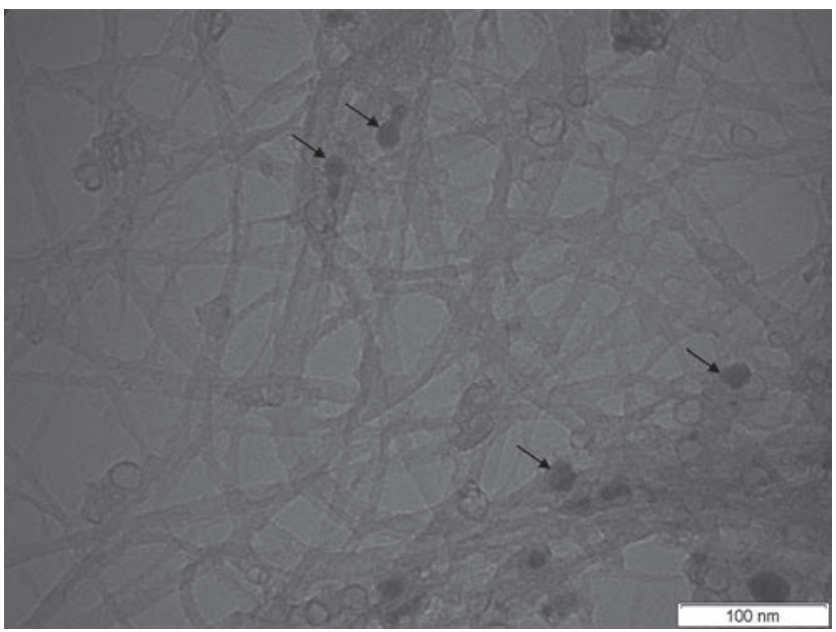

Fig. 2 Transmission electron microscopyimage of original single-wall carbon nanotubes. Arrows show the presence of impurities, mainly amorphous carbon and metal nanoparticles.

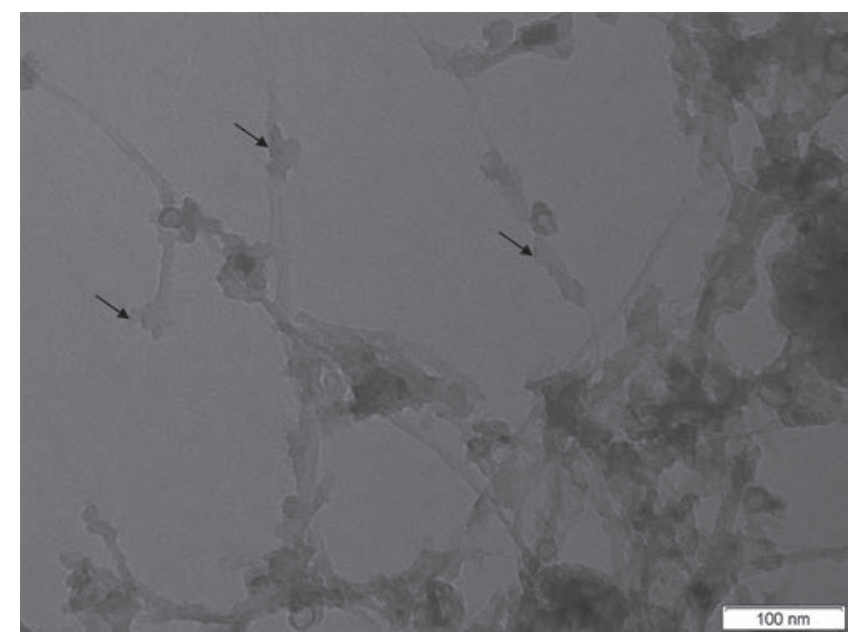

Fig. 3 Transmission electron microscopy image of SWCNTs after silanization (SWCNTs/SiO 2 -ATES). The nanotubes appear scattered and the arrows show the growth of nano-particles of silicon oxides adhered to their surfaces. SWCNT, single-wall carbon nanotubes. 
value of flexural strength obtained was probably caused by a SWCNTs agglomeration, leading to less energy dissipating in the system under viscoelastic deformation. Another explanation may be related to the opacity of the SWCNTs agglomerates. This could have interfered with the degree of conversion of monomers and consequently affected the properties of the composite material. It is more important to have dispersion and an accurate load transfer between nano-reinforcement and matrix than to increase the content of nanotubes. ${ }^{24}$ However, when these values were compared to those from composite without nanotubes (CG), the flexural strength of the SWCNTs/SiO2-ATES was consistently lower. In the present study, purified SWCNTs with carboxylic sites were used, and the presence of carboxylic acid groups on the nanotube surface facilitates further functionalization because this group can participate in a variety of chemical reactions. ${ }^{25}$ The organosilane ATES, used in this study, supports the combination between $\mathrm{Si}-\mathrm{OH}$ and $\mathrm{OH}$ - of the surface of SWCNTs while the organic portion can react with the composite monomer via the $\mathrm{C}=\mathrm{C}$ group. ${ }^{23}$

When comparing the results of the present study to Zhang et al, ${ }^{22}$ some differences can be found. The authors incorporated a silanized SWCNTs, in a weight ratio of 0.1 (SWCNTs: paste), in a composite paste (Durafill; Heraeus-Kulzer, Germany) and reported that the specimens containing modified SWCNTs exhibit considerable improvement in flexural strength compared to Durafill without SWCNTs. This contradictory finding may be attributed to the differing methodologies and composite employed. The authors incorporated a silanized SWCNTs in a weight ratio of 0.1 in a composite paste, using a blended monomer of urethane dimethacrylate. A low viscosity of a resin enables particles to be organized within the resin.

The indirect composite system Sinfony is a microhybrid material that was developed to be applied using the layering technique because of its flow consistence. In this study, the SWCNTs were spatulated directly with the resin paste. This process could have affected the properties of the resin where a large amount of filler had been inserted in an inadequate quantity of organic matrix, resulting in a decrease of the flexural strength of Sinfony resin modified by SWCNTs. Nanotubes must be uniformly dispersed as isolated nanotubes individually coated with polymer.

In other study, authors reported that composites including silanated nanofiller, as opposed to untreated nanofiller, not only presented superior mechanical properties, but were more resistant to water sorption and general wear. ${ }^{26}$ These finds are in agreement with our study where SWCNTs/SiO2-ATES presents higher flexural strength than SWCNTs/0.1, SWCNTs/0.2, and SWCNTs/0.3 groups that are not silanized. Moreover, TEM images of SWCNTs after silanization showed the presence of growth of nanoparticles of silicon oxides adhered to the surfaces ( - Fig. 2). Despite these analysis have confirmed that SWCNTs were silanized, the process utilized might not be so effective because CG present higher values of flexural strength when compared with SWCNTs/SiO2-ATES group, suggesting that the SWCNTs act as weakness area where there is a poor chemical bonding between the nanofiller and Sinfony organic phase. This hypothesis may also be suggested for SWCNTs/SiO2-ATES when compared with SWCNTs/0.1; SWCNTs/0.2, and SWCNTs/0.3 groups. Within the limitation of this study, the results showed that the incorporation of CNT could be an interesting procedure to improve dental composites flexural strength; however, this incorporation should be performed with caution, since the amount of CNT can interfere in the wettability of the resin matrix and the degree of conversion. Further studies should investigate different surface treatments of CNTs and the effects of its incorporation on a dental composite.

\section{Conclusion}

The silanization process improves the SWCNTs strength proprieties, but the modification of chemical bonding between SWCNT and SWCNT/SiO 2 -ATES modified resins, in different concentrations, did not improve the composite resin flexural strength. Since RBC restorations are widely used, the clinical indications of each type of resin composite are dictated by the amount of stresses applied on the restoration as well as the esthetic requirements. Therefore, understanding how CNT reinforces dental composites can be an alternative to create a stronger and cheap material that could be used in the dental office in cases of fixed partial dentures.

\section{Funding}

This study received its funding from the Coordination of Superior Level Staff Improvement, (finance code 001) and the National Council for Scientific and Technological Development.

\section{Conflict of Interest}

None declared.

\section{References}

1 Suzuki S, Nagai E, Taira Y, Minesaki Y. In vitro wear of indirect composite restoratives. J Prosthet Dent 2002;88(4):431-436

2 Jordan RE, Suzuki M. Posterior composite restorations. Where and how they work best. J Am Dent Assoc 1991;122(11):30-37

3 Lacy AM. A critical look at posterior composite restorations. J Am Dent Assoc 1987;114(3):357-362

4 Manhart J, Chen H, Hamm G, Hickel R. Buonocore Memorial Lecture. Review of the clinical survival of direct and indirect restorations in posterior teeth of the permanent dentition. Oper Dent 2004;29(5):481-508

5 Wilder ADJ, Bayne SC, Heymann HO. Long-term clinical performance of direct posterior composites. Trans Acad Dent Mater 1996;9(33):151-169

$6 \mathrm{Kim} \mathrm{KH}$, Ong JL, Okuno O. The effect of filler loading and morphology on the mechanical properties of contemporary composites. J Prosthet Dent 2002;87(6):642-649

7 Ellakwa AE, Shortall AC, Marquis PM. Influence of fiber type and wetting agent on the flexural properties of an indirect fiber reinforced composite. J Prosthet Dent 2002;88(5):485-490

8 Freilich MA, Duncan JP, Meiers JC, Goldberg AJ. Preimpregnated, fiber-reinforced prostheses. Part I. Basic rationale and complete-coverage and intracoronal fixed partial denture designs. Quintessence Int 1998;29(11, 11Pt 1) :689-696 
9 Ausman KD, Piner R, Lourie O, Ruoff RS, Korobov M. Organic solvent dispersions of single-walled carbon nanotubes: toward solutions of pristine Nanotubes. J Phys Chem B 2000;104(38):8911-8915

10 Lourie O, Wagner HD. Transmission electron microscopy observations of fracture of single-wall carbon nanotubes under axial tension. Appl Phys Lett 1998;73(24):3527-3529

11 Schadler LS, Giannaris SC, Ajayan PM. Load transfer in carbon nanotube epoxy composites. Appl Phys Lett 1998;73(26):3842-3844

12 Behr M, Rosentritt M, Latzel D, Kreisler T. Comparison of three types of fiber-reinforced composite molar crowns on their fracture resistance and marginal adaptation. J Dent 2001;29(3):187-196

13 Lehmann F, Eickemeyer G, Rammelsberg P. Fracture resistance of metal-free composite crowns-effects of fiber reinforcement, thermal cycling, and cementation technique. J Prosthet Dent 2004;92(3):258-264

14 Brunton PA, Cattell P, Burke FJ, Wilson NH. Fracture resistance of teeth restored with onlays of three contemporary tooth-colored resin-bonded restorative materials. J Prosthet Dent 1999;82(2):167-171

15 Ellakwa A, Thomas GD, Shortall AC, Marquis PM, Burke FJ. Fracture resistance of fiber-reinforced composite crown restorations. Am J Dent 2003;16(6):375-380

16 Moniruzzaman M, Winey KL. Polymer nanocomposites containing carbon nanotubes. Macromolecules 2006;39(16):5194-5195

17 Dasa A, Stöckelhubera KW, Jurka R, et al. Modified and unmodified multiwalled carbon nanotubes in high performance solution-styrene-butadiene and butadiene rubber blends. Polymer (Guildf) 2008;49(24):5276-5283
18 Gong X, Liu J, Baskaran S, Voise RD, Young JS. Surfactant-assisted processing of carbon nanotube/polymer composites. Chem Mater 2000;12(4):1049-1052

19 Xie XL, Maia YW, Zhou XP. Dispersion and alignment of carbon nanotubes in polymer matrix: A review. Mater Sci Eng 2005;49(4):89-112

20 Wanga YP, Chenga RL, Lianga LL, Wang YM. Study on the preparation and characterization of ultra-high molecular weight polyethylene-carbon nanotubes composite fiber. Compos Sci Technol 2005;65(5):793-797

21 Krithikadatta J, Gopikrishna V, Datta M. CRIS Guidelines (checklist for reporting in-vitro studies): a concept note on the need for standardized guidelines for improving quality and transparency in reporting in-vitro studies in experimental dental research. J Conserv Dent 2014;17(4):301-304

22 Zhang F, Xia Y, Xu L, Gu N. Surface modification and microstructure of single-walled carbon nanotubes for dental resin-based composites. J Biomed Mater Res B Appl Biomater 2008;86(1):90-97

23 Xia Y, Zhang F, Xie H, Gu N. Nanoparticle-reinforced resin-based dental composites. J Dent 2008;36(6):450-455

24 Prolongo SG, Gude MR, Ureña A. Synthesis and characterisation of epoxy resins reinforced with carbon nanotubes and nanofibers. J Nanosci Nanotechnol 2009;9(10):6181-6187

$25 \mathrm{Xu} \mathrm{HH}$, Quinn JB, Giuseppetti AA, Eichmiller FC, Parry EE, Schumacher GE. Three-body wear of dental resin composites reinforced with silica-fused whiskers. Dent Mater 2004;20(3):220-227

26 Terry DA. Direct applications of a nanocomposite resin system: Part 1-the evolution of contemporary composite materials. Pract Proced Aesthet Dent 2004;16(6):417-422 